\title{
DIAGNÓSTICO E INTERVENÇÕES À PESSOA COM TRANSTORNO MENTAL COM BASE NA CONSULTA DE ENFERMAGEM
}

Danielly de Souza Canabrava', Tatiana Brusamarello² ${ }^{2}$ Fernanda Carolina Capistrano ${ }^{3}$, Verônica de Azevedo Mazza ${ }^{4}$, Nen Nalú Alves das Mercês ${ }^{5}$, Mariluci Alves Maftum ${ }^{6}$

RESUMO: O estudo objetivou identificar diagnósticos de enfermagem e elaborar plano de cuidado à pessoas com transtorno mental. Trata-se de uma pesquisa de prática assistencial desenvolvida de março a junho de 2008 com 10 pacientes de uma unidade de internação integral feminina, em hospital especializado em psiquiatria situado em um município do Paraná. Os dados foram obtidos por meio da consulta de enfermagem. Da análise, interpretação e julgamento clínico das respostas apresentadas foram obtidos 13 diagnósticos e 57 intervenções de enfermagem, organizados segundo o modelo de estrutura da taxonomia proposto por North American Nursing Diagnosis Association. Foi possível por em prática um agir terapêutico e perceber a possibilidade de elaborar intervenções de enfermagem com a participação do paciente. As atividades e responsabilidades inerentes ao enfermeiro tornaram-se explícitas, bem como a importância da consulta de enfermagem no processo de trabalho.

PALAVRAS-CHAVE: Saúde mental; Enfermagem; Diagnóstico de enfermagem.

\section{DIAGNOSIS AND INTERVENTIONS FOR THE PERSON WITH MENTAL DISORDERS BASED ON THE NURSING CONSULTATION}

ABSTRACT: The study aimed to identify nursing diagnoses and elaborate care plans for people with mental disorders. This is care practice research undertaken in March-June 2008 with 10 patients from a female acute psychiatric treatment unit, in a specialized psychiatric hospital in a municipality in Paraná. The data was obtained from the nursing consultations. From the analysis, interpretation and judgment, 13 diagnoses and 57 nursing interventions were obtained, organized according to the model for structuring taxonomy proposed by the North American Nursing Diagnosis Association. It was possible to put therapeutic action into practice and perceive the possibility of elaborating nursing interventions with the client's participation. The nurse's activities and responsibilities become explicit, as well as the importance of the nursing consultation in the work process.

KEYWORDS: Mental health; Nursing; Nursing diagnosis.

\section{DIAGNÓSTICO E INTERVENCIONES A LA PERSONA CON TRASTORNO MENTAL CON BASE EN LA CONSULTA DE ENFERMERÍA}

RESUMEN: El estudio tuvo la finalidad de identificar diagnósticos de enfermería y elaborar plan de cuidado a personas con transtorno mental. Es una investigación de práctica asistencial desarrollada de marzo a junio de 2008 con 10 pacientes de una unidad de internación integral femenina, en hospital especializado en psiquiatría ubicado en un municipio del Paraná. Los datos fueron obtenidos por medio de consulta de enfermería. Del análisis, interpretación y juzgamiento clínico de las respuestas presentadas fueron obtenidos13 diagnósticos y 57 intervenciones de enfermería, organizados segúnel modelo de estructura de taxonomía propuesto por North American Nursing Diagnosis Association. Fue posible poner en práctica un actuar terapéutico y percibir la posibilidad de elaborar intervenciones de enfermería con la participación del paciente. Las actividades y responsabilidades inherentes al enfermero se mostraron explícitas así como la importancia de la consulta de enfermería en el proceso de trabajo.

PALABRAS CLAVE: Salud mental; Enfermería; Diagnóstico de enfermería.

${ }^{1}$ Enfermeira.

${ }^{2}$ Enfermeira. Mestranda pelo Programa de Pós-Graduação em Enfermagem da Universidade Federal do Paraná - PPGENF UFPR. Membro do Núcleo de Estudos, Pesquisa e Extensão em Cuidado Humano em Enfermagem - NEPECHE.

${ }^{3}$ Acadêmica do Curso de Graduação em Enfermagem da UFPR. Membro do NEPECHE. Bolsista CNPq de Iniciação Científica.

${ }^{4}$ Enfermeira. Doutora em Enfermagem. Professora do Departamento e do PPGENF UFPR. Membro do Grupo de Estudo da Família, Saúde e Desenvolvimento - GEFASED.

${ }^{5}$ Enfermeira. Doutora em Enfermagem. Professora do Departamento e do PPGENF UFPR. Membro do NEPECHE.

${ }^{6}$ Enfermeira. Doutora em Enfermagem. Professora do Departamento e do PPGENF UFPR. Coordenadora do PPGENF UFPR. Vice-líder do NEPECHE. 


\section{INTRODUÇÃO}

A Enfermagem no decorrer de sua história acumulou conhecimentos empíricos e, na maioria das vezes, seus profissionais têm realizado as ações baseadas em normas e rotinas repetidas e também atuado sem uma reflexão da sua prática, mesmo com as modificações do perfil da clientela, do avanço tecnológico e da organização dos próprios profissionais de enfermagem. Entretanto, a utilização de um método de trabalho que favoreça a individualização e a continuidade do cuidado de enfermagem e estimule o pensamento crítico do enfermeiro é uma estratégia competente para minimizar esse problema ${ }^{(1)}$.

Desenvolver o processo de enfermagem indica que o enfermeiro está realizando um trabalho profissional específico com a adoção de um método fundamentado em um sistema de valores e crenças morais e em conhecimento técnico-científico da área em que atua ${ }^{(2)}$.

A etapa do processo de enfermagem, conhecida como coleta de dados, através do histórico e exame físico na consulta de enfermagem, favorece a aproximação do paciente. Para obter resultados significativos, o enfermeiro deve ser capaz de detectar com precisão os problemas ou as reações humanas que evidenciam desequilíbrios, distinguindo com exatidão as variações e o grau de comprometimento para a saúde de cada indivíduo ${ }^{(1)}$.

Ressalta-se a importância da consulta de enfermagem em saúde mental, pois ela enseja trabalhar sintomas clínicos do transtorno mental, considerados primários, como delírios e alucinações, como também os sintomas secundários como a solidão, isolamento social e problemas afetivos que, por vezes, são relegados a um segundo plano, não sendo identificados como problemas de enfermagem ${ }^{(3)}$.

A consulta de enfermagem proporciona, sistematicamente, o levantamento dos problemas e das reações humanas, permitindo o conhecimento do histórico de saúde do paciente e da família. Indo além dos aspectos biológicos, o diagnóstico de enfermagem, o planejamento, a implementação de ações, a avaliação do cuidado e das condições de saúde com orientações influenciam na adoção de práticas favoráveis à saú$\mathrm{de}^{(4)}$. A credibilidade sobre a prática de enfermagem repousa, principalmente, sobre as ações resultantes de diagnósticos estabelecidos a partir do julgamento sobre as necessidades humanas específicas, visando a atingir um resultado pelo qual o profissional é legalmente responsável ${ }^{(2)}$.
A utilização dos diagnósticos de enfermagem possibilita a definição de ações que vão intervir no processo de vida e de saúde-doença dos indivíduos, quando são alcançados os resultados pelos quais o enfermeiro é responsável. Também permite o uso de uma linguagem única e padronizada, favorecendo o processo de comunicação, o planejamento de ações, a elaboração de prescrições e intervenções, o desenvolvimento de pesquisas e o processo de ensino-aprendizagem profissional, conferindo cientificidade ao cuidado ${ }^{(5)}$.

De acordo com a North American Nursing Diagnosis Association (NANDA), diagnóstico de enfermagem é "[...] um julgamento clínico sobre as respostas do indivíduo, da família ou da comunidade a problemas de saúde/processos vitais reais ou potenciais"(6:293). Este diagnóstico, por sua vez, confere a base para que o enfermeiro realize as prescrições de enfermagem e, consequentemente, para os resultados dos cuidados prestados ao paciente. A identificação dessa prática aumenta a responsabilidade dos enfermeiros ao avaliar os pacientes, determinar os diagnósticos e proporcionar as intervenções adequadas a ele $^{(6)}$.

A prescrição de enfermagem é definida como qualquer intervenção e/ou cuidado direto a ser realizado em benefício do paciente e, quanto mais informações forem obtidas sobre o indivíduo, melhor será a qualidade do cuidado desenvolvido. Os diagnósticos e as intervenções serão melhor elaborados e mais adequados aos dados coletados, e a avaliação das respostas do paciente à terapêutica implementada será muito mais segura e confiável( ${ }^{(7)}$.

Portanto, este estudo de prática assistencial mediante a estratégia de consulta de enfermagem teve como objetivo identificar diagnósticos de enfermagem e elaborar um plano de cuidados a pessoas com transtorno mental.

\section{MÉTODO}

Pesquisa qualitativa desenvolvida no período de março a junho de 2008. O método utilizado foi a pesquisa prática, compreendida como aquela ligada à práxis e que tem o propósito de intervir na realidade social. É a investigação científica conduzida para gerar conhecimento, influenciando diretamente a prática assistencial, com o objetivo de resolver problemas específicos, tomar decisões ou controlar consequências de situações da vida real ${ }^{(8)}$.

A pesquisa foi desenvolvida em uma unidade de internação integral feminina com capacidade 
para 30 pacientes, de um Hospital da rede pública estadual, especializado em Psiquiatria, situado em um município do Paraná.

Os dados foram obtidos por meio da Consulta de Enfermagem realizada com dez pacientes. Durante a consulta, foram levantados os diagnósticos de enfermagem padronizados de acordo com a taxonomia do NANDA. Posteriormente, foi elaborado um plano de cuidados de acordo com as intervenções de enfermagem para cada paciente. As intervenções foram aplicadas após a análise do plano de cuidado.

Os pacientes com transtorno mental são considerados grupo vulnerável por estarem com sua autonomia comprometida no momento da internação. Eles participaram da consulta de enfermagem, tendo respeitados seus direitos, e assinaram o Termo de Consentimento Livre e Esclarecido de acordo com a Resolução 196/96(9). Para garantir o sigilo e o anonimato, os sujeitos foram identificados por algarismos arábicos, como, por exemplo, Sujeito S1, S2, etc. Os dados foram organizados em quadros e são apresentados de modo descritivo.

Esta pesquisa foi aprovada pelo Comitê de Ética e Pesquisa do Setor de Ciências da Saúde da Universidade Federal do Paraná - UFPR, CEP/SD 461.141.07.12.

\section{RESULTADOS}

Das dez participantes: sete tinham entre 17 e 38 anos; três, entre 39 e 60 anos; cinco estavam separadas; três solteiras; uma casada; e uma estava divorciada. Os diagnósticos clínicos foram (7) Transtornos Afetivos bipolar, com episódio atual maníaco com sintomas psicóticos, (2) com Esquizofrenia paranoide e (1) com diagnóstico de Transtorno comportamental devido ao uso de múltiplas drogas. Em relação ao número de internações, havia uma paciente com dez internações, uma paciente com nove internações, três pacientes com seis internações, duas pacientes com três internações e três pacientes com duas internações.

A interpretação dos dados obtidos e o julgamento clínico das respostas verbais e comportamentais apresentadas pelas pacientes durante as Consultas de Enfermagem aos problemas de saúde vivenciados permitiram identificar 13 diagnósticos de enfermagem (Quadros 1, 2, 3 e 4). Para estes diagnósticos, foram elaboradas 57 intervenções, sendo três no mínimo por diagnóstico, criadas considerando as ações inerentes ao fazer; ajudar, orientar, supervisionar e encaminhar. Cada paciente recebeu entre cinco e 10 diagnósticos, tendo alguns destes diagnósticos se repetido entre as pacientes.
A aplicação das intervenções para cada paciente foi contínua durante as interações diárias. Algumas intervenções foram realizadas em grupo, pois envolviam várias pacientes com os mesmos diagnósticos de enfermagem. Essas intervenções ocorreram nos encontros realizados na sala de reunião da unidade de internação, com exceção das caminhadas, que aconteceram no pátio interno. Cada encontro durou em média 20 minutos e aconteceu uma vez por semana.

Quadro 1 - Diagnósticos e intervenções de enfermagem relativos ao domínio funcional de acordo com classificação da NANDA. Curitiba, 2008

\begin{tabular}{|c|c|}
\hline \multicolumn{2}{|c|}{ 1.Domínio Funcional } \\
\hline $\begin{array}{l}\text { Diagnóstico de enfer- } \\
\text { magem }\end{array}$ & $\begin{array}{l}\text { Intervenção de enferma- } \\
\text { gem }\end{array}$ \\
\hline \multicolumn{2}{|c|}{ Classe: Atividade/Exercício } \\
\hline $\begin{array}{l}\text { 1. Estilo de vida seden- } \\
\text { tário (Todos os partici- } \\
\text { pantes) }\end{array}$ & $\begin{array}{l}\text { 1. Estimular a participa- } \\
\text { ção nas atividades físicas. } \\
\text { 2. Proporcionar cami- } \\
\text { nhadas. } \\
\text { 3. Incentivar a deambu- } \\
\text { lação. }\end{array}$ \\
\hline \multicolumn{2}{|c|}{ Classe: Autocuidado } \\
\hline $\begin{array}{l}\text { 2. Déficit no autocuidado } \\
\text { para banho/higiene (To- } \\
\text { dos os sujeitos) }\end{array}$ & $\begin{array}{l}\text { 4. Orientar cuidados com } \\
\text { a pele. } \\
\text { 5. Orientar como deve ser } \\
\text { feita a higiene corporal } \\
\text { e oral. } \\
\text { 6. Incentivar o autocui- } \\
\text { dado. } \\
\text { 7. Incentivar a valoriza- } \\
\text { ção do "Eu". } \\
\text { 8. Incentivar a participa- } \\
\text { ção nas atividades gru- } \\
\text { pais sobre autocuidado. }\end{array}$ \\
\hline \multicolumn{2}{|c|}{ Classe: Sono e Repouso } \\
\hline $\begin{array}{l}\text { 3. Privação de sono } \\
\text { (Participantes } 1,5,7,8 \text {, } \\
9,10)\end{array}$ & $\begin{array}{l}\text { 9. Atentar para alteração } \\
\text { de humor ou comporta- } \\
\text { mento. } \\
\text { 10. Observar quantidade } \\
\text { de horas dormidas. } \\
\text { 11. Atentar para reações } \\
\text { adversas à medicação. } \\
\text { 12. Estimular participa- } \\
\text { ção nas atividades pro- } \\
\text { postas. } \\
\text { 13. Estimular a saída do } \\
\text { leito durante o dia. }\end{array}$ \\
\hline
\end{tabular}


Quadro 2 - Diagnósticos e intervenções de enfermagem relativos ao domínio fisiológico de acordo com classificação da NANDA. Curitiba, 2008

\begin{tabular}{|c|c|}
\hline \multicolumn{2}{|c|}{$\begin{array}{l}\text { 2.Domínio Fisiológico } \\
\end{array}$} \\
\hline $\begin{array}{l}\text { Diagnóstico de enfer- } \\
\text { magem }\end{array}$ & Prescrição de enfermagem \\
\hline \multicolumn{2}{|c|}{ Classe: Integridade tissular } \\
\hline $\begin{array}{l}\text { 4. Mucosa oral prejudi- } \\
\text { cada (Participantes } 1,3 \text {, } \\
4,5,7,8,9,10)\end{array}$ & $\begin{array}{l}\text { 14. Estimular aumento da } \\
\text { ingesta hídrica. } \\
\text { 15. Hidratar os lábios com } \\
\text { lubrificante aquoso. } \\
\text { 16. Incentivar bochecho } \\
\text { com água. }\end{array}$ \\
\hline \multicolumn{2}{|c|}{ Classe: Líquido e eletrólitos } \\
\hline $\begin{array}{l}\text { 5. Volume de líquidos } \\
\text { deficiente (Participantes } \\
2,3,4,5,6,7,9)\end{array}$ & $\begin{array}{l}\text { 17. Observar a quantidade } \\
\text { de líquido ingerido. } \\
\text { 18. Verificar sinais de res- } \\
\text { secamento na pele. } \\
\text { 19. Orientar para que co- } \\
\text { munique a equipe de saúde } \\
\text { quando o débito urinário } \\
\text { estiver alterado. }\end{array}$ \\
\hline \multicolumn{2}{|c|}{ Classe: Eliminação } \\
\hline $\begin{array}{l}\text { 6. Constipação } \\
(\text { Participantes } 1,2,3, \\
5,6,9)\end{array}$ & $\begin{array}{l}\text { 20. Estimular ingesta de } \\
\text { alimentos ricos em fibras. } \\
\text { 21. Incentivar a ingesta } \\
\text { hídrica. } \\
\text { 22. Auscultar ruídos hidro- } \\
\text { aéreos. } \\
\text { 23. Observar tempo de } \\
\text { constipação. } \\
\text { 24. Incentivar prática de } \\
\text { exercícios físicos. }\end{array}$ \\
\hline \multicolumn{2}{|c|}{ Classe: Neurocognição } \\
\hline $\begin{array}{l}\text { 7. Processo de pensa- } \\
\text { mento perturbado (Par- } \\
\text { ticipantes } 1,3,4,5,6,7, \\
8,9,10)\end{array}$ & $\begin{array}{l}\text { 25. Auxiliar na interpreta- } \\
\text { ção precisa do ambiente. } \\
\text { 26. Auxiliar a identificar o } \\
\text { real e o imaginário. } \\
\text { 27. Trazer a paciente à } \\
\text { realidade. } \\
\text { 28. Observar hipovigilân- } \\
\text { cia e hipervigilância. } \\
\text { 29. Abordar a paciente com } \\
\text { tranquilidade e respeito. } \\
\text { 30. Identificar foco da an- } \\
\text { siedade ou preocupação. } \\
\text { 31. Orientar a paciente } \\
\text { sobre seu papel no trata- } \\
\text { mento. }\end{array}$ \\
\hline
\end{tabular}

Quadro 3 - Diagnósticos e intervenções de enfermagem relativos ao domínio psicossocial de acordo com a classificação da NANDA. Curitiba, 2008

\begin{tabular}{|c|c|}
\hline \multicolumn{2}{|c|}{$\begin{array}{l}\text { 3.Domínio Psicossocial } \\
\end{array}$} \\
\hline $\begin{array}{l}\text { Diagnóstico de } \\
\text { enfermagem }\end{array}$ & Prescrição de enfermagem \\
\hline \multicolumn{2}{|c|}{ Classe: Enfrentamento } \\
\hline $\begin{array}{l}\text { 8. Enfrenta- } \\
\text { mento ineficaz } \\
\text { (Todos os Par- } \\
\text { ticipantes) }\end{array}$ & $\begin{array}{l}\text { 32. Incentivar a avaliar seu próprio } \\
\text { comportamento. } \\
\text { 33. Mobilizar para um aumento } \\
\text { gradual das atividades. } \\
\text { 34. Investigar a capacidade de rela- } \\
\text { tar os fatos. } \\
\text { 35. Incentivar o relacionamento } \\
\text { interpessoal. }\end{array}$ \\
\hline $\begin{array}{l}\text { 9. En frenta- } \\
\text { mento Familiar } \\
\text { comprometido } \\
\text { (Participantes } \\
2,5,8,10)\end{array}$ & $\begin{array}{l}\text { 36. Incentivar a aproximação da } \\
\text { família com a paciente. } \\
\text { 37. Ressaltar a importância da fa- } \\
\text { mília no tratamento. } \\
\text { 38. Valorizar suas atitudes positivas. } \\
\text { 39. Orientar sobre a melhor maneira } \\
\text { de se cuidar. }\end{array}$ \\
\hline \multicolumn{2}{|r|}{ Classe: Comportamento } \\
\hline $\begin{array}{l}\text { 10. Manuten- } \\
\text { ção ineficaz da } \\
\text { saúde (Partici- } \\
\text { pantes } 1,3,4,5 \text {, } \\
6,7,9,10)\end{array}$ & $\begin{array}{l}\text { 40. Estimular interessa na melhora } \\
\text { comportamental. } \\
\text { 41. Identificar grau de prejuízo } \\
\text { cognitivo. } \\
\text { 42. Potencializar conhecimentos de } \\
\text { controle pessoal, orientando no reco- } \\
\text { nhecimento dos sintomas individuais. } \\
\text { 43. Estabelecer plano para diminuir o } \\
\text { número de cigarros fumados por dia. } \\
\text { 44.Identificar as situações que a } \\
\text { levam a fumar. } \\
\text { 45. Discutir os malefícios do tabaco. }\end{array}$ \\
\hline $\begin{array}{l}11 \text {. Controle } \\
\text { do regime te- } \\
\text { rapêutico fa- } \\
\text { miliar ineficaz } \\
\text { (Participantes } \\
1,3,4,5,7,8,9)\end{array}$ & $\begin{array}{l}\text { 46. Orientar a família sobre necessi- } \\
\text { dade de ter alguém responsável por } \\
\text { administrar as medicações em casa. } \\
\text { 47. Orientar sobre a necessidade de } \\
\text { respeitar a paciente. } \\
\text { 48. Esclarecer dúvidas sobre doença } \\
\text { do familiar internado. }\end{array}$ \\
\hline & Classe: Emocional \\
\hline $\begin{array}{l}\text { 12. Ansiedade } \\
\text { (Participantes } \\
1,2,3,5,7,8, \\
9,10)\end{array}$ & $\begin{array}{l}\text { 49. Observar insônia. } \\
\text { 50. Esclarecerdúvidas sobreotratamento. } \\
\text { 51. Identificar o foco da ansiedade. } \\
\text { 52. Estabelecercontatoverbal terapêutico. } \\
\text { 53. Observar alterações fisiológicas. } \\
\text { 54. Observar sinais vitais. }\end{array}$ \\
\hline
\end{tabular}


Quadro 4 - Diagnósticos e intervenções de enfermagem relativos ao domínio meio ambiente de acordo com classificação da NANDA. Curitiba, 2008

\begin{tabular}{|c|c|}
\hline \multicolumn{2}{|c|}{ 4.Domínio Meio ambiente } \\
\hline $\begin{array}{l}\text { Diagnóstico de enfer- } \\
\text { magem }\end{array}$ & $\begin{array}{l}\text { Prescrição de enferma- } \\
\text { gem }\end{array}$ \\
\hline \multicolumn{2}{|c|}{ Classe: Controle de riscos } \\
\hline $\begin{array}{l}\text { 13. Manutenção do lar } \\
\text { prejudicada (Participan- } \\
\text { tes } 3,6,7,8,10 \text { ) }\end{array}$ & $\begin{array}{l}\text { 55. Incentivar a participação } \\
\text { nas atividades de manuten- } \\
\text { ção e limpeza da unidade. } \\
\text { 56. Orientar sobre a im- } \\
\text { portância do ambiente } \\
\text { limpo } \\
\text { 57. Reabilitar a paciente } \\
\text { nas atividades diárias. }\end{array}$ \\
\hline
\end{tabular}

\section{DISCUSSÃO}

A faixa etária das participantes da pesquisa está na idade considerada de maior produtividade em relação ao mundo do trabalho e nas questões que envolvem a concepção e cuidado de filhos. Esses dados corroboram a existência de forte associação entre os fatores biológicos predisponentes e os fatores psicológicos relacionados com a mulher na sociedade moderna. Esta enfrenta sobrecarga de trabalho, problemas familiares, profissionais, financeiros, entre outros, que culminam nessa faixa etária levando, por vezes, à instalação de um quadro de transtorno mental ${ }^{(10)}$.

Das cinco participantes que referiram estar separadas de seus cônjuges, três relataram que a separação aconteceu antes do surgimento do primeiro episódio de transtorno mental e duas apresentaram o transtorno durante o período em que estavam casadas e, segundo elas, o transtorno mental contribuiu para a separação conjugal.

Acredita-se que a alta taxa de reinternação esteja relacionada também com o diagnóstico médico, sendo predominante o Transtorno Afetivo bipolar ${ }^{(7)}$. As taxas de não-adesão ao tratamento são altas para as pessoas com transtorno bipolar, representando $47 \%$ em alguma fase do tratamento ou $52 \%$ durante um período de dois anos. Isso explica também o fato de as pacientes estarem em fase de mania, pois as taxas de não-adesão podem aumentar a recorrência de mania, haja vista que $60 \%$ das pacientes internadas com mania aguda apresentavam falhas no uso da medicação no mês que antecedeu a sua hospitalização ${ }^{(10)}$.

É relevante ressaltar que para se chegar a um diagnóstico de enfermagem é necessário levar em conta um processo sistematizado, considerando as características definidoras e os fatores relacionados. A estrutura da taxonomia do NANDA ${ }^{(6)}$ compreende três níveis para definição do diagnóstico - domínios, classes e diagnósticos de enfermagem - que foram agrupados nos Quadros 1, 2, 3 e 4.

Vale destacar que domínio é "uma esfera de atividade, estudo ou interesse"; classe é "uma subdivisão de um grupo maior, uma divisão de pessoas ou coisas por qualidade, grau ou categoria"; e o diagnóstico é a base para a seleção das intervenções de enfermagem e alcance dos resultados esperados ${ }^{(6: 293)}$.

Com relação ao domínio funcional, apresentado no quadro 1 , todas as participantes do estudo tiveram pelo menos um ou mais diagnósticos de enfermagem em diferentes classes. O estilo de vida sedentário pode relacionar-se com a medicação utilizada no tratamento dos transtornos mentais. Um dos efeitos desejáveis e/ ou colaterais é a sedação, que influencia na atividade física. Outro aspecto é a fisiopatologia da doença, com sintomas que influenciam a diminuição da atividade física, como anedonia, alterações da consciência, delírios de ruína, entre outros. Como intervenção de enfermagem, foram organizados momentos de atividade física com caminhadas e alongamento no pátio interno da unidade ${ }^{(7)}$.

$\mathrm{O}$ autocuidado engloba as atividades necessárias para a satisfação das necessidades diárias, que são aprendidas ao longo do tempo e se tornam hábitos de vida. $\mathrm{O}$ motivo de estabelecer este diagnóstico para um paciente pode estar relacionado ao prejuízo na função motora ou cognitiva, causando uma diminuição na capacidade de desempenhar as atividades de autocuidado ${ }^{(7)}$.

Todas as pacientes apresentaram este diagnóstico e foram promovidas atividades semanais em grupo, como palestra educativa com material lúdico e discussão em roda de conversa a respeito da importância da higiene corporal. Em alguns casos foi prescrito o auxílio na higienização para a paciente fazê-la sozinha.

O diagnóstico de enfermagem de privação do sono relaciona-se com o estado em que o indivíduo apresenta uma mudança na quantidade ou na qualidade do seu padrão de repouso, causando desconforto ou interferindo no seu estilo de vida desejável. Essas perturbações do sono podem ser resultantes de modificações ou problemas fisiológicos, psicológicos, sociais e ambientais $^{(7)}$. As intervenções de enfermagem foram mais relacionadas à equipe de enfermagem em observar as pacientes, proporcionar um ambiente tranquilo durante a noite e estimular a paciente a sair do leito durante 
o dia. Foram também dadas orientações às pacientes com relação ao benefício do sono e discussão dos casos mais graves com a equipe multiprofissional.

No domínio fisiológico, quadro 2, foi apresentado o diagnóstico de enfermagem "Mucosa oral prejudicada", estado em que a pessoa apresenta lesões na cavidade oral, podendo estar relacionadas com irritantes químicos como drogas, álcool e tabaco. As causas mais comuns estão relacionadas com a falta de higiene oral e efeitos colaterais das medicações como as fenotiazinas e anticolinérgicos que diminuem a salivação ${ }^{(7)}$. Ao identificar os fatores relacionados a este diagnóstico em cada paciente, foi definida a intervenção de enfermagem, constituída por orientação, auxílio e supervisão da higienização oral. Nos casos relacionados com a medicação, foi estimulado aumentar a ingesta hídrica.

O referente diagnóstico "Volume de líquidos deficiente", está relacionado à menor motivação para ingerir líquidos. Nestes casos, a intervenção de enfermagem foi organizar quatro vezes ao dia a ingestão de líquidos em grupo e reforçar a importância dessa atitude durante as palestras educativas e rodas de conversa $^{(7)}$. A constipação resulta da passagem atrasada de resíduos alimentares, que, no caso das pacientes do estudo, está relacionada ao estresse, falta de exercícios físicos, ingestão inadequada de líquido e efeitos colaterais pela utilização de fenotiazinas ${ }^{(7)}$. Em alguns desses fatores, a enfermeira pode intervir conforme descrito nas intervenções dos diagnósticos de estilo de vida sedentário e volume de líquidos deficientes. Também a enfermeira pode discutir com a equipe multiprofissional e com um nutricionista, para modificar a dieta do paciente, dependendo do caso.

No diagnóstico de "Processos de pensamento perturbados", em que as pacientes apresentam alteração na percepção e na cognição interferindo nas atividades cotidianas, as causas mais comuns são os distúrbios psicológicos, como, por exemplo, a depressão, transtornos de personalidade e $\operatorname{bipolar}^{(7)}$. A enfermagem, para intervir, precisa focar na redução do pensamento, transformando-o e ou promovendo orientação para a realidade. Para exemplificar as intervenções relativas a este diagnóstico de enfermagem, foram promovidas rodas de conversa, interações fundamentadas na comunicação terapêutica.

A comunicação é um instrumento de enfermagem de grande importância, pois permite ao profissional estabelecer um relacionamento com os pacientes a fim de auxiliá-los a suprir suas necessidades em relação à saúde. Para tanto, é imprescindível que o profissional de enfermagem estabeleça e mantenha uma comunicação eficaz com o paciente a fim de criar vínculos para um relacionamento baseado na confiança e segurança, para que a pessoa com transtorno mental possa falar de si e de seus problemas com segurança e tranquilidade, depositando credibilidade na assistência de enfermagem ${ }^{(11)}$.

Referente aos diferentes domínios psicossocial apresentados no quadro 3, o diagnóstico "Enfrentamento ineficaz" é definido como o

estado em que o indivíduo apresenta ou está em risco de apresentar incapacidade para controlar adequadamente os estressores internos ou ambientais devido a recursos inadequados (físicos, psicológicos, comportamentais e/ou cognitivos) $)^{(7: 231)}$.

O diagnóstico de enfermagem "Manutenção ineficaz da saúde" é apropriado para pessoas que expressam o desejo de modificar o estilo de vida pouco saudável. A enfermagem deve estimular e reforçar as atitudes que $\mathrm{o}$ paciente apresenta com o objetivo de melhorar sua saúde ${ }^{(7)}$.

O diagnóstico de enfermagem "Ansiedade" envolve um sentimento vago de apreensão e intranquilidade em resposta à ameaça aos sistemas e ao valor ou ao padrão de segurança da pessoa. Os sinais e sintomas de ansiedade que podemos destacar são: tensão aumentada, agitação geral, insônia, preocupação, sentimento de desamparo, entre outros ${ }^{(7)}$. As pessoas e as famílias submetidas a diversos problemas de saúde, agudos ou crônicos, enfrentam geralmente programas de tratamento que exigem mudanças no funcionamento e no estilo de vida anterior. Essas mudanças ou adaptações podem ser instrumentos que influenciam os resultados positivos. Quando a pessoa enfrenta um regime complexo a ser seguido ou um funcionamento comprometido impedindo o controle, bem como o sucesso do tratamento, o estabelecimento do diagnóstico "Controle familiar ineficaz do regime terapêutico" é apropriado( ${ }^{(7,12)}$.

Para o diagnóstico "Enfrentamento familiar comprometido", a família é vista como um sistema com interdependência entre seus membros, assim, os desafios da vida para os membros individuais também atingem o sistema familiar. Certas situações têm o potencial de influenciar negativamente o funcionamento familiar, e os exemplos incluem hospitalização e crise econômica, entre outros ${ }^{(7,12)}$.

Em todos os diagnósticos relacionados anteriormente, a intervenção foi essencialmente baseada nas relações interpessoais, tanto individual como grupal, de acordo com a competência da enfermeira. Quando 
o diagnóstico estava relacionado com a família, a intervenção era por meio do repasse das informações para a Assistente Social e a Psicóloga, que faziam atendimento familiar semanalmente.

No domínio "Meio ambiente", apresentado no quadro 4, foi identificado como diagnóstico de enfermagem a "Manutenção do lar prejudicada", configurada por situações nas quais a pessoa necessita de ensino, supervisão e ou assistência para controlar a manutenção doméstica. Nesse estudo, somente foi possível trabalhar com o ensino, uma vez que a paciente não se encontrava em seu ambiente familiar ${ }^{(7)}$. Algumas intervenções foram realizadas coletivamente por meio de atividades lúdicas, palestras educativas ou em roda de conversa. As atividades realizadas em conjunto permitem a troca de vivências e experiências entre os pacientes, trazendo inúmeros benefícios além de atingirem uma grande quantidade de paciente que possuem o mesmo diagnóstico de enfermagem, potencializando as ações da enfermeira.

Após as intervenções com as pacientes, era realizado o registro da evolução de enfermagem individual. A evolução de enfermagem de cada paciente possibilita determinar os resultados da implementação das intervenções de enfermagem e, com isto, incluir, modificar ou suspender os cuidados considerados pertinentes ${ }^{(7)}$.

\section{CONCLUSÕES}

Durante este estudo, as atividades e responsabilidades inerentes ao enfermeiro tornaram-se explícitas bem como a importância da consulta de enfermagem no processo de trabalho. Reafirmou-se que a enfermagem possui um caráter relacional e que o relacionamento interpessoal é complexo visto que são pessoas em interação, cada uma delas com suas características pessoais, sociais e culturais.

Foi possível por em prática o agir de modo terapêutico, que consiste em observar, ouvir, perceber a comunicação não verbal e procurar compreender os acontecimentos que envolvem o cuidar. A consulta de enfermagem facilitou a aproximação e a liberdade de diálogo entre enfermeiro e paciente com desenvolvimento de vínculos de confiança potencializados pelos momentos de interações durante as outras atividades de enfermagem.

Percebeu-se que durante a consulta de enfermagem foi possível esboçar estratégias de ajuda e elaborar prescrições de enfermagem com a participação da própria paciente. Contudo, nos problemas que necessitam de atendimento especializado, era esclarecido que seria feito um encaminhamento, pois existem casos que o enfermeiro está impossibilitado de resolver, como, por exemplo, perda de um ente querido, em que eram realizados trabalhos relativos à esperança.

Destaca-se que novos estudos devem ser desenvolvidos a fim de contribuir na efetiva aplicação da consulta de enfermagem bem como para uma futura implementação de tal prática, fundamental no processo de trabalho do enfermeiro, proporcionando assim um cuidar reflexivo, com qualidade e visibilidade profissionais.

\section{REFERÊNCIAS}

1. Zagonel, IPS. Consulta de enfermagem: um modelo de metodologia para o cuidado. In: Westphalen MEA, Carraro TE, organizadores. Metodologias para assistência de enfermagem: teorização, modelos e subsídios para a prática. Goiânia: AB; 2001. p. 41-56.

2. Garcia TR, Nóbrega MML. Processo de enfermagem: da teoria à prática assistencial e de pesquisa. Esc. Anna Nery. [Internet] 2009;13(1) [acesso em 20 out 2011]. Disponível: http://www.scielo.br/pdf/ean/v13n1/ v13n1a26.pdf

3. Campos JCL, Oliveira RMP, Casanova EG, Cardoso MMVN. Reabilitação psicossocial e enfermagem psiquiátrica. In: Figueiredo NMA, Machado WCA, Tonini T, organizadores. Cuidando de clientes com necessidades especiais, motora e social. São Paulo: Difusão; 2004. p. 205-16.

4. Mendes MH, Freitas VA, Gomes ET. Consulta de enfermagem: uma prática necessária aos indivíduos com transtornos mentais. Revista Enfermagem Integrada. [Internet] 2009;2(1) [acesso em 20 out 2011]. Disponível: http://www.unilestemg.br/enfermagemintegrada/artigo/ v2/Mariana_mendes_Valeria_freitas_e_Everton_ gomes.pdf

5. Truppel TC, Meier MJ, Calixto RC, Peruzzo AS, Crozeta K. Sistematização da Assistência de Enfermagem em unidade de terapia intensiva. Rev Bras Enferm. [Internet] 2009;62(2) [acesso em $10 \mathrm{dez} 2011$ ]. Disponível: http://www.scielo.br/pdf/reben/v62n2/ a08v62n2.pdf

6. North American Nursing Diagnosis Association (NANDA). Diagnósticos de enfermagem da NANDA: definições e classificação 2007-2008. Trad. de Regina Machado Garcez. Porto Alegre: Artmed; 2008.

7. Carpenito LJ. Diagnósticos de enfermagem: aplicação 
à prática clínica. 13 ${ }^{\mathrm{a}}$ ed. Porto Alegre: Artmed; 2012.

8. Demo P. Metodologia do conhecimento científico. São Paulo: Atlas; 2000.

9. Ministério da Saúde(BR). Conselho Nacional de Saúde. Diretrizes e normas regulamentadoras de pesquisa envolvendo seres humanos. Resolução n. 196, de 10 de outubro de 1996. Brasília; 1996.

10. Pegoraro RF, Caldana RHL. Mulheres, loucura e cuidado: a condição da mulher na provisão e demanda por cuidados em saúde mental. Saude soc. [Internet] 2008;17(2) [acesso em $10 \mathrm{dez} 2011$ ]. Disponível: http://www.scielo.br/scielo.php?pid=S010412902008000200009\&script $=$ sci_arttext

11. Girade MG, Cruz EMNT, Stefanelli MC. Educação continuada em enfermagem psiquiátrica: reflexão sobre conceitos. Rev Esc Enferm USP. [Internet] 2006;40(1) [acesso em 19 mar 2012]. Disponível: http://www.scielo.br/scielo.php?script=sci_arttext\&pi $\mathrm{d}=\mathrm{S} 0080-62342006000100015$

12. Gentile C, Pereira MA. O. A doença mental: visão de pacientes psicóticos. Cogitare enferm. [Internet] 2005;10(2) [acesso em 19 mar 2012]. Disponível: http://ojs.c3sl.ufpr.br/ojs2/index.php/cogitare/article/ view/5007/3785 